\title{
Mobile Phone Antenna with Reduced Radiation into Inner Ear
}

\author{
Jamal S. Rahhal \\ Electrical Engineering Department, The University of Jordan, Amman, Jordan \\ Email: rahhal@ju.edu.jo
}

Received 19 September 2014; revised 25 October 2014; accepted 7 November 2014

Copyright (C) 2014 by author and Scientific Research Publishing Inc.

This work is licensed under the Creative Commons Attribution International License (CC BY).

http://creativecommons.org/licenses/by/4.0/

(c) (i) Open Access

\begin{abstract}
Health hazards are of great concern to cellular phone users. One important measure of the effect of electromagnetic radiation into human body is the specific absorption rate (SAR). If the human body is exposed to electromagnetic radiation, the amount of power absorbed by its tissues per mass volume should be limited and not to exceed a maximum SAR value. The cellular phone radiated through its antenna in all directions a certain amount of electromagnetic energy. This energy is concentrated in the near field region, where the user's head is located during the call. The closest organ that is very sensitive to temperature change is the inner ear (it is just under the cellular phone antenna) where it contains a controlled viscosity liquid. In this paper we devise a twoantenna design to generate a low radiation in the direction of user's head while using the cellular phone. By creating a null in the radiation pattern in the direction of user's head we minimize the risk of hazards on the user. We optimize the null steering such that the device maintains a good connection to its base station and keeps the SAR level under the allowed maximum value using Lagrange method. To implement the analytical solution in real time simulated annealing (SA) algorithm is used. Results showed that we could steer the radiation pattern to optimize the radiated power in the direction of base station under the limited SAR level constraint. Simulated annealing algorithm is adopted to find the near optimal delay value to steer the antenna radiation pattern since it finds the global optimal point. It shows that a real time processing on the mobile unit can be performed to solve for the best null direction while the device is active.
\end{abstract}

\section{Keywords}

Electromagnetic Radiation, SAR, Patch Antenna, EM, Health Hazards, Lagrange Multiplier and Simulated Annealing

\section{Introduction}

The design of antennas for wireless personal communication systems is the subject of much research that is mo- 
tivated by size, efficiency and health issues. In addition to maximizing the antenna radiated/accepted power of the handsets, the effects on the antenna performance from surrounding objects such as the human body must be considered. On the other hand the effect of radiation on the human body must be also considered. The closest human sensitive part to the handset in calling position is the human brain and ear in most mobile models or at least close enough to cause harmful effects. The tissues of these organs are mostly nerves plus liquid and hence, they carry electrical signals that might be affected by electromagnetic radiation from the wireless device [1]-[9].

The RF energy is scattered and attenuated as it propagates through the tissues of the head, and maximum energy absorption is expected in the more absorptive high water-content tissues near the surface of the head. Inner ear (that contains high water-content) is just under the mobile phone and it will be subject to the strongest radiation from the mobile unit as shown in Figure 1.

The electromagnetic (EM) penetration into human head causes permanent damage to tissues that are exposed to high density EM energy. This could cause some organs to malfunction or at least a disturbance in their functionality. The amount of exposed energy that can be handled by human tissues is measured by the specific absorption rate (SAR) that is given by [10]-[15]:

$$
\mathrm{SAR}=\frac{\sigma|E|^{2}}{2 \rho} \mathrm{W} / \mathrm{kg}
$$

where $E$ is the electric field intensity, $\sigma$ is the tissue conductivity and $\rho$ is the tissue density. The dependency of $\sigma$ on frequency is the result of interaction between the EM waves and the tissue material, such that the existence of ions will increase the conductivity and will change the permittivity of the tissue. The complex nature of the permittivity reflected into changing the conductivity of the tissue.

The effect of radiation in the inner ear has two folds: the effect on the neural tissues (hearing) and the effect on the filling liquid (balance). The radiation devices must be compliant to the SAR standard IEEE C95.1. The IEEE exposure criteria are based on a determination that potentially harmful biological effects can occur at an SAR level of $4 \mathrm{~W} / \mathrm{kg}$ as averaged over the whole-body. Appropriate safety factors were then added to arrive at limits for both whole-body exposure $(0.4 \mathrm{~W} / \mathrm{kg}$ for "controlled" or "occupational" exposure and $0.08 \mathrm{~W} / \mathrm{kg}$ for "uncontrolled" or "general population" exposure, respectively) and for partial-body (localized SAR), this might occur in the head of the user of a hand-held cellular telephone [9].

The nature of the tissues in the inner ear makes its relative dielectric permittivity in the order of $(41.5+j 17.98$ at $900 \mathrm{MHz}$ and $40.0+j 13.98$ at $1.8 \mathrm{GHz}$ ) and its conductivity $(0.97$ at $900 \mathrm{MHz}$ and 1.4 at $1.8 \mathrm{GHz})$. The problem with the inner ear arises from the fact that its inner liquid heat dissipation is not suited to dissipate heat generated from high radiation near the ear. This will maximize the risk of losing balance and/or changing the physical characteristics of the inner ear and hence, hearing impairment might occur [10] [12].

This paper presents an antenna design to minimize radiation in the inner ear direction and at the same time produce an acceptable radiation pattern that can be used to communicate with base stations.

\section{Antenna System Description}

The proposed antenna system in this paper consists of ground plane and two H shaped PCB tracks on the other side that is using a coaxial feeder as shown in Figure 2. The radiation pattern for each antenna in the near field and the far field are shown in Figure 3 and Figure 4. The combination of the two antenna elements makes it possible to steer the radiation pattern toward the base station and creates a null toward the human head. This will

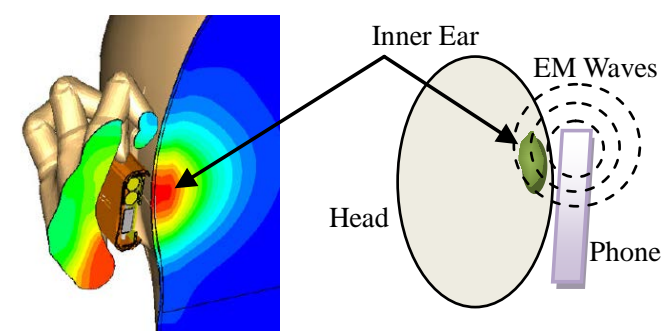

Figure 1. Mobile phone radiation into human head showing the inner ear location. 


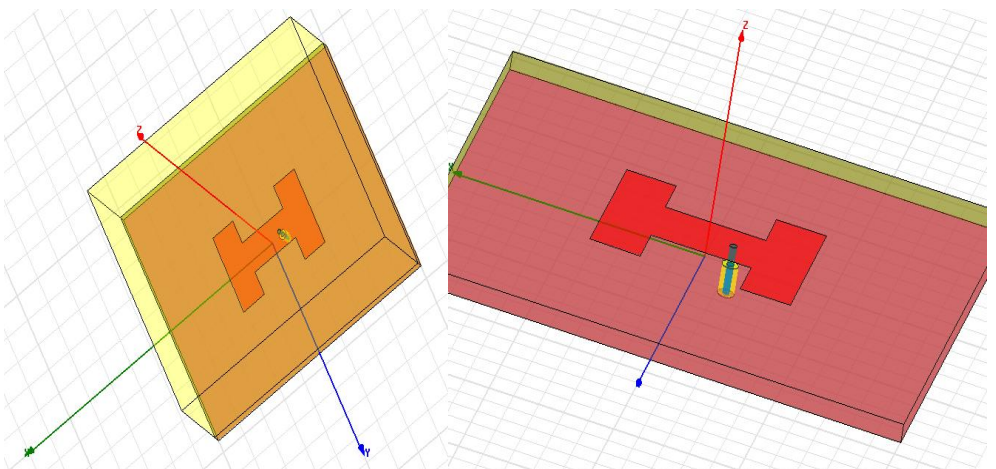

Figure 2. One element antenna geometry.
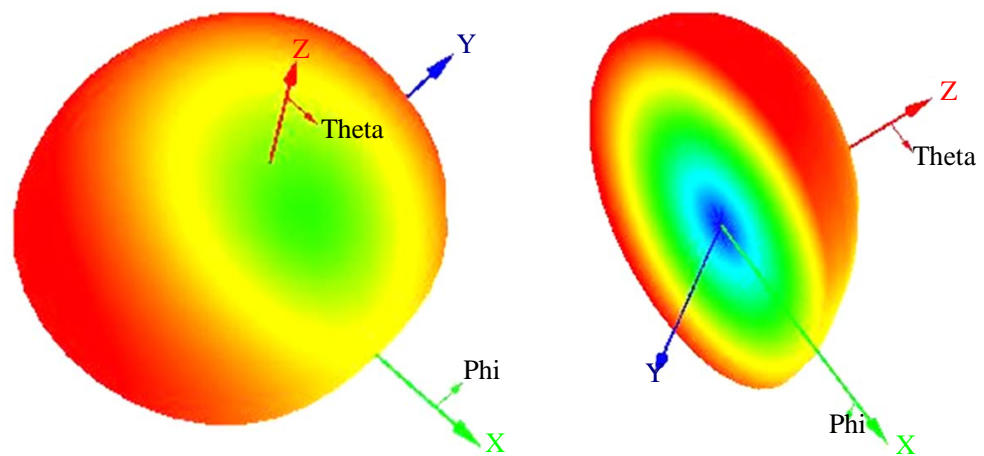

Figure 3. Near field radiation pattern for one antenna element; (showing half space).
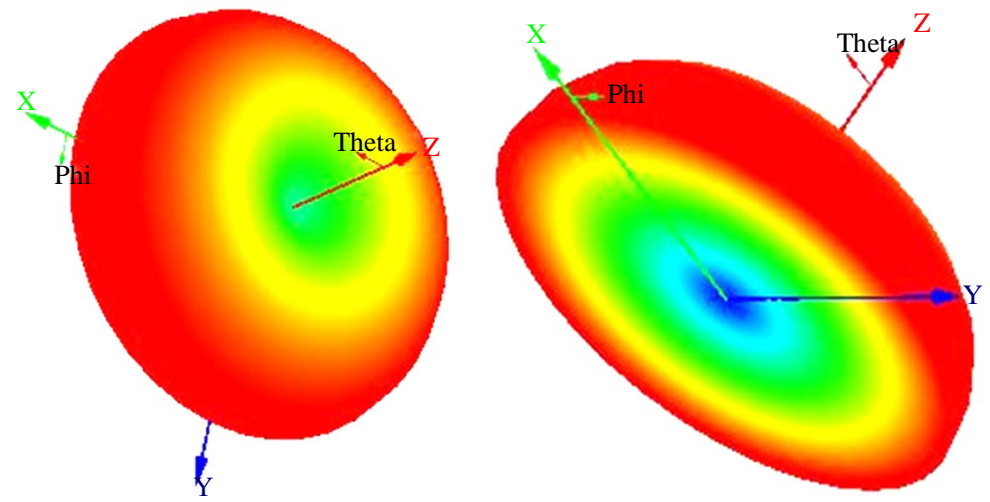

Figure 4. Far field radiation pattern for one antenna element; (showing half space).

reduce the SAR in the human head and maintains the communication with the base station.

The two antenna elements proposed here will have an $\mathrm{H}$ shape each as shown in Figure 5. The near field radiation pattern for each element as seen from Figure 3 is close to Omni directional. Therefore, the electric field can be expressed in mathematical form as [16]-[18]:

$$
E(x, y, z)=E_{o}
$$

where $E(x, y, z)$ is the electric field radiated from the antenna in the near field and $E_{o}$ is constant in all directions.

To steer the radiation pattern, a variable delay element is introduced in the feeding circuit of one of the elements. Assuming that it is required to steer the radiation pattern main beam in $\theta$ direction and steer the null is 


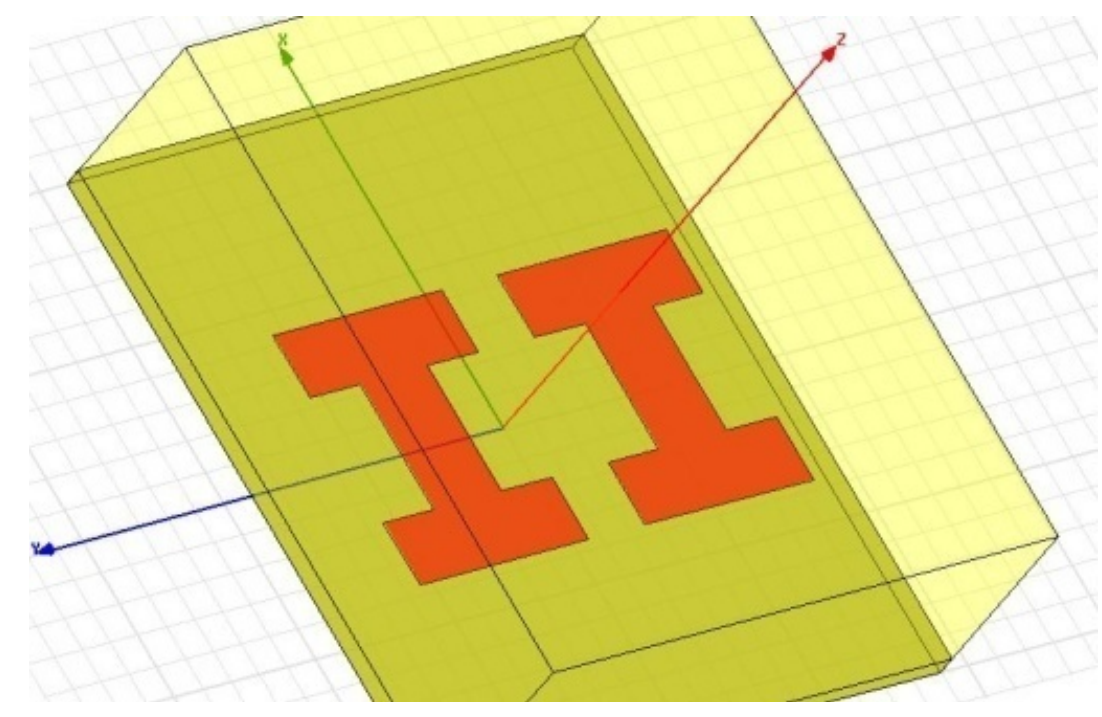

Figure 5. The two elements antenna geometry.

$\beta$ direction (that is usually in the normal direction to the front plane of the cellular phone) then the delay $\tau$ is found by:

$$
\tau=\frac{T \delta}{2 \pi}
$$

where $\delta$ is the equivalent phase shift in radians and $T$ is the carrier period. To find the optimal $\delta$ that maximises the radiation pattern in the base station direction and at the same time minimize the radiation pattern in the human head direction to maintain acceptable SAR value, we solve the following equations:

$$
E_{h}=E_{o}\left(1+\mathrm{e}^{j(\beta-\delta)}\right)
$$

And

$$
\mathrm{SAR}=\frac{\sigma E_{o}^{2}\left|1+\mathrm{e}^{j(\beta-\delta)}\right|^{2}}{2 \rho}
$$

The electric field to the base station direction is given by:

$$
E_{r}=E_{o}\left(1+\mathrm{e}^{j(\theta-\delta)}\right)
$$

Then to find the optimal $\delta$ we maximize:

$$
\frac{P_{r}}{\mathrm{SAR}}=K \frac{\left|1+\mathrm{e}^{j(\theta-\delta)}\right|^{2}}{\left|1+\mathrm{e}^{j(\beta-\delta)}\right|^{2}}
$$

where $P_{r}$ is the received power from base station and $K$ is a constant. This equation is represented graphically as shown in Figure 6 and it can be maximized analytically.

For example if $\theta=45^{\circ}$ and $\beta=-20^{\circ}$ in the $y$-z plane. Here the optimal $\delta$ has more than one optimal value $\delta \in\left\{-85^{\circ},-42^{\circ}, 3^{\circ}, 47^{\circ}, 90^{\circ}\right\}$ as shown in Figure 7 . The radiation pattern in the near field for the two elements at $\delta=3^{\circ}$ is shown in Figure 8.

The above example shows that there are several solutions for Equation (7). This is due to the existence of nulls in the dominator of the equation. As both $\theta$ and $\beta$ get close to each other the optimization becomes less efficient and the maximum value for $P_{r} / \mathrm{SAR}$ becomes less for example for $\theta=45^{\circ}$ and $\beta=40^{\circ}$ the optimal $\delta$ has more than one optimal value $\delta \in\left\{-70^{\circ},-25^{\circ}, 20^{\circ}, 65^{\circ}\right\}$ as shown in Figure 9. 


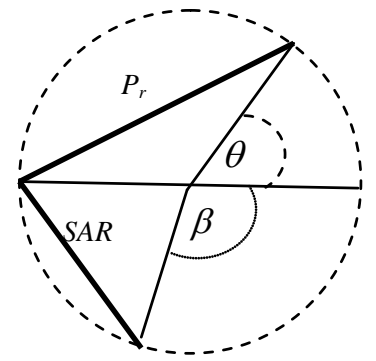

Figure 6. The geometrical representation of the performance equation.

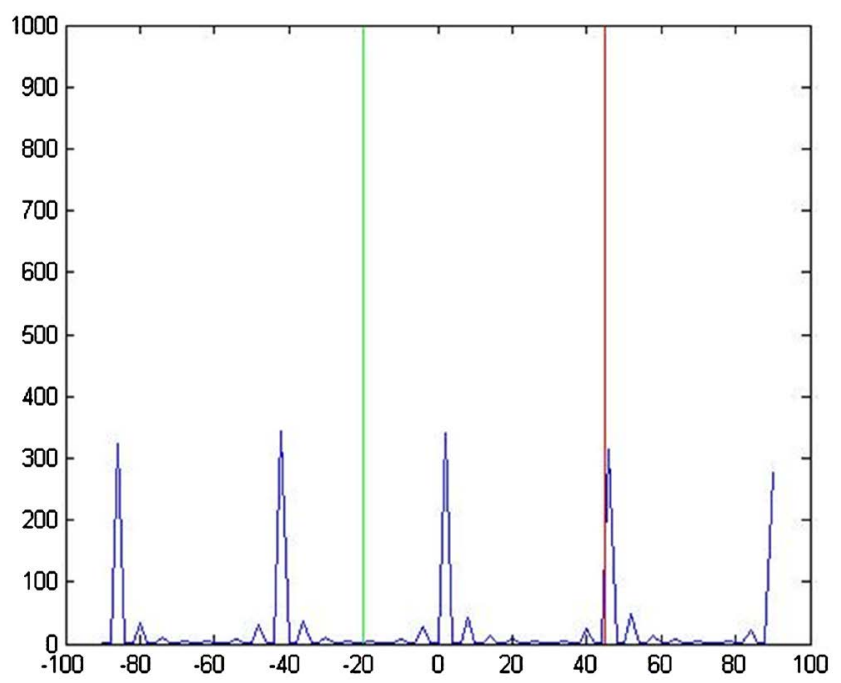

Figure 7. $P_{r} / \mathrm{SAR}$ vs the phase shift $\delta$ for $\theta=45^{\circ}$ and $\beta=-20^{\circ}$.

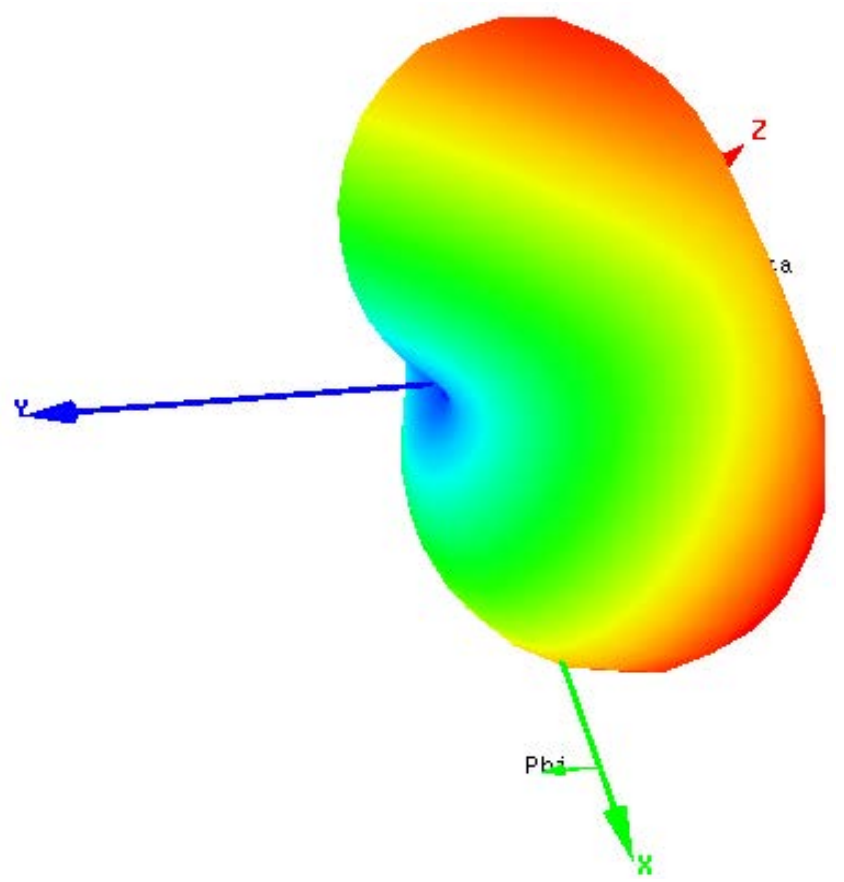

Figure 8. Near field radiation pattern for two antenna elements. 


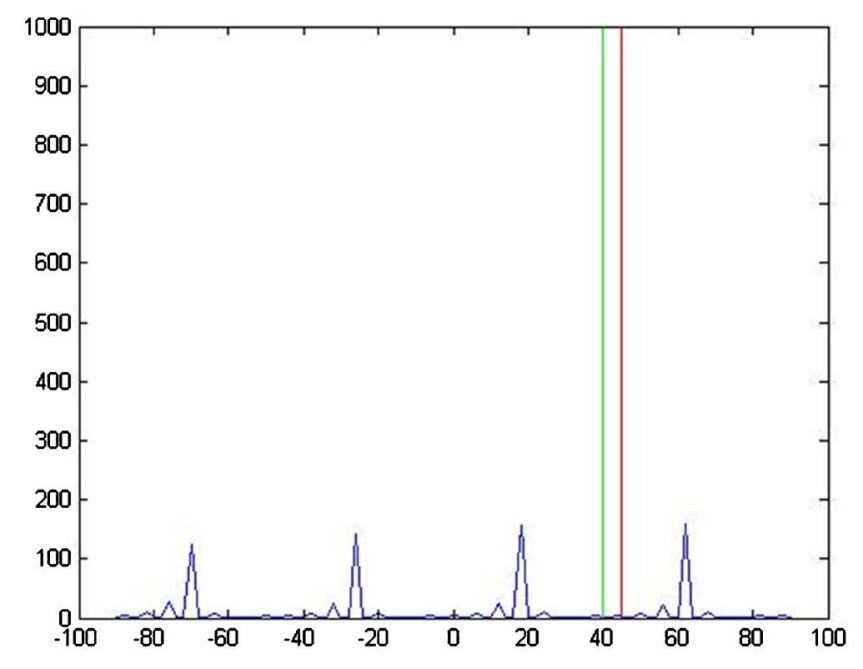

Figure 9. $P_{r} / \mathrm{SAR}$ vs the phase shift $\delta$ for $\theta=45^{\circ}$ and

$$
\beta=40^{\circ} \text {. }
$$

Direct maximization of Equation (7) yields to solution where the SAR in the dominator of the equation is very small and hence any value for the electric field in the base station direction will maximize the equation. This happens when $\beta-\delta \cong n \pi$ and any value of the $E_{r}$ will maximize $P_{r} /$ SAR. To solve this problem, we use a constraint optimization technique, such that we impose the constraint not to exceed a certain value of SAR and maximize the received power in the direction of the base station. Lagrange multiplier method can be used to find the optimal value of $\delta$, such that we maximize the radiated energy in the base station direction under the constraint not to exceed a maximum value for the SAR. The cost function can be written as:

$$
C=\max \left\{\left(\left|1+\mathrm{e}^{j(\theta-\delta)}\right|^{2}\right)+\lambda\left(\frac{\sigma}{2 \rho}\left|1+\mathrm{e}^{j(\beta-\delta)}\right|^{2}-\mathrm{SAR}\right)\right\}
$$

where $\lambda$ is the Lagrange multiplier.

$$
\frac{\partial C}{\partial \delta}=\left[-2 \sin (\theta-\delta)-2 \lambda \frac{\sigma}{2 \rho} \sin (\beta-\delta)\right]=0
$$

Solving for $\lambda$ we find that:

$$
\lambda=-\frac{2 \rho \sin (\theta-\delta)}{\sigma \sin (\beta-\delta)}
$$

And from the constraint:

$$
\cos (\beta-\delta)+1=\frac{\rho \mathrm{SAR}}{\sigma}
$$

From Equation (10) we find that:

$$
\delta=\theta+\sin ^{-1}\left(\frac{\lambda \sigma}{2 \rho} \sin (\beta-\delta)\right) \rightarrow \delta=g(\lambda)
$$

The function $g(\lambda)$ is assumed here since analytical result is hard to get. A good approximation yields to:

$$
\delta \cong\left(\frac{\theta+\frac{\lambda \sigma}{2 \rho} \beta}{1+\frac{\lambda \sigma}{2 \rho}}\right)
$$


Solving for $\delta$ as a function of $\lambda$ and substituting in Equation (11). Then finding the value of $\lambda$ from Equation (11) that satisfies the constraint and substituting it in Equation (10) to get the optimal value of $\delta_{\text {opt }}$.

$$
\lambda=g^{-1}\left(\beta-\cos ^{-1}\left(\frac{\rho \mathrm{SAR}}{\sigma}-1\right)\right)
$$

And:

$$
g^{-1}\left(\beta-\cos ^{-1}\left(\frac{\rho \mathrm{SAR}}{\sigma}-1\right)\right)=-\frac{2 \rho \sin \left(\theta-\delta_{\mathrm{opt}}\right)}{\sigma \sin \left(\beta-\delta_{\mathrm{opt}}\right)}
$$

Note that the analytical solution is hard to get since Equations (10) and (11) are not linear. We need a numerical technique to find $\delta_{\text {opt }}$ such that, the solution can be found fast and the mobile device can determine the optimal delay in real time. We propose to use an iterative technique based on simulated annealing (SA) method to solve for the optimal delay [19]-[22]. This technique will result in a sub-optimal value for $\delta_{\text {opt }}$ but it should converge to a solution in real time. Equation (10) has more than one solution depending on the values of $\theta$ and $\beta$, some of them are local optimal values. We need to find the global optimal value, and therefore, simulated annealing algorithm is selected since it converges to the global optimal point (or near optimal). Starting from the cost function defined as:

$$
F=\hat{\delta}_{\text {opt }}-h\left(\delta_{\text {opt }}, \beta, \theta, \rho, \sigma, \mathrm{SAR}\right)
$$

An approximation of this cost function is given by:

$$
F=\frac{2 \rho \sin \left(\theta-\delta_{\mathrm{opt}}\right)}{\sigma \sin \left(\beta-\delta_{\mathrm{opt}}\right)}-\left(\frac{\frac{\sigma}{2 \rho} \cos ^{-1}\left(\frac{\rho \mathrm{SAR}}{\sigma}-1\right)}{\beta-\theta-\cos ^{-1}\left(\frac{\rho \mathrm{SAR}}{\sigma}-1\right)}\right)
$$

The devised system need to know the base station direction as well as the SAR direction $(\beta, \theta)$, these angels should be known each optimization update. The SAR angle is easy to obtain since it is always normal to the speaker of the phone as shown in Figure 10. $\theta$ is usually unknown and need to be estimated on real time. To estimate the arrival angle many techniques may be used. Here we may use simple method to measure $\theta$, such that, by using the received signal strength indicator (RSSI) of the device when on receiving mode and sweeping the delay between the elements to get maximum RSSI.

The simulated annealing algorithm does not require derivative information; it needs to be supplied with a cost function for each trial solution it generates. The algorithm simulates a small random displacement of an atom that results in a change in energy. If the change in energy is negative, the energy state of the new configuration is lower and the new configuration is accepted. If the change in energy is positive, the new configuration has a higher energy state; however, it may still be accepted according to the Boltzmann probability factor given by:

$$
P=\mathrm{e}^{-\frac{\Delta E}{k_{b} T}}
$$

where $k_{b}$ is the Boltzmann constant, $T$ is the current temperature and $\Delta E$ is the change in energy (cost

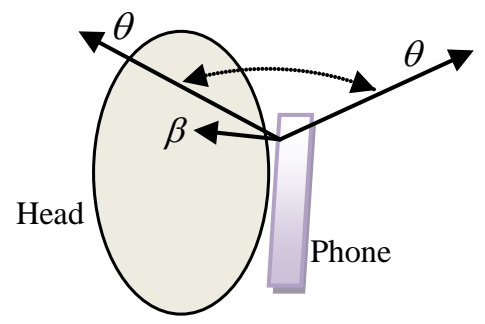

Figure 10. Usual expected directions for $\theta$ and $\beta$. 
function). The solution is started at a high "temperature", where it has a high cost. Random perturbations are then made to the solution. If the cost is lower, the new solution is made the current solution; if it is higher, it may still be accepted according the probability given by the Boltzmann factor. The Boltzmann probability is compared to a random number drawn from a uniform distribution between 0 and 1 ; if the random number is smaller than the Boltzmann probability, the solution is accepted. This allows the algorithm to escape local minima. As the temperature is gradually lowered, the probability that a worse solution is accepted becomes smaller. Although the algorithm is not guaranteed to find the best optimum, it will often find near optimum and it is also a simple algorithm to implement.

The simulated annealing algorithm is given as in the following pseudo code:

\begin{tabular}{|cl|}
\hline $\mathrm{s} \leftarrow \mathrm{s}_{0} ; \mathrm{e} \leftarrow \mathrm{E}(\mathrm{s})$ & // Initial solution, energy. \\
$s_{\text {best } \leftarrow \mathrm{s} ; e_{\text {best }} \leftarrow \mathrm{e}}$ & // Initial "best” solution. \\
$\mathrm{k} \leftarrow 0$ & // Energy evaluation count. \\
while $\mathrm{k}<\mathrm{k}_{\max }$ and $\mathrm{e}>\mathrm{e}_{\max }$ & // Loop: \\
$\mathrm{T} \leftarrow$ temperature $\left(\mathrm{k} / \mathrm{k}_{\max }\right)$ & // Temperature calculation. \\
$s_{\text {new }} \leftarrow$ neighbour $(\mathrm{s})$ & // Pick some neighbour. \\
$e_{\text {new }} \leftarrow \mathrm{E}\left(s_{\text {new }}\right)$ & // Compute its energy. \\
if $P\left(e, e_{\text {new }}, T\right)>$ random( $)$ then & // Check if should we use it. \\
$s \leftarrow s_{\text {new }} ; e \leftarrow e_{\text {new }}$ & // Change state. \\
if $e_{\text {new }}<e_{\text {best }}$ then & // A new best? \\
$s_{\text {best }} \leftarrow s_{\text {new }} ; e_{\text {best }} \leftarrow e_{\text {new }}$ & // Save “new neighbour" to "best found". \\
$\mathrm{k} \leftarrow \mathrm{k}+1$ & // loop. \\
return $s_{\text {best }}$ & // Return the best solution found. \\
\hline
\end{tabular}

In the following we use MatLab to calculate the optimal delay for the previous examples using the simulated annealing algorithm.

\section{Numerical Calculation and Results}

To demonstrate the performance of the devised system and to find the optimal delay value using simulated annealing algorithm we use MatLab software to find the optimal delay for the examples discussed in the previous section: In the first example where $\theta=45^{\circ}$ and $\beta=-20^{\circ}$. Figure 11 shows a numerical calculation of the cost function given in Equation (17). Here the optimal $\delta$ has more than one solution at the zero crossing points one of them is the global minimum cost solution.

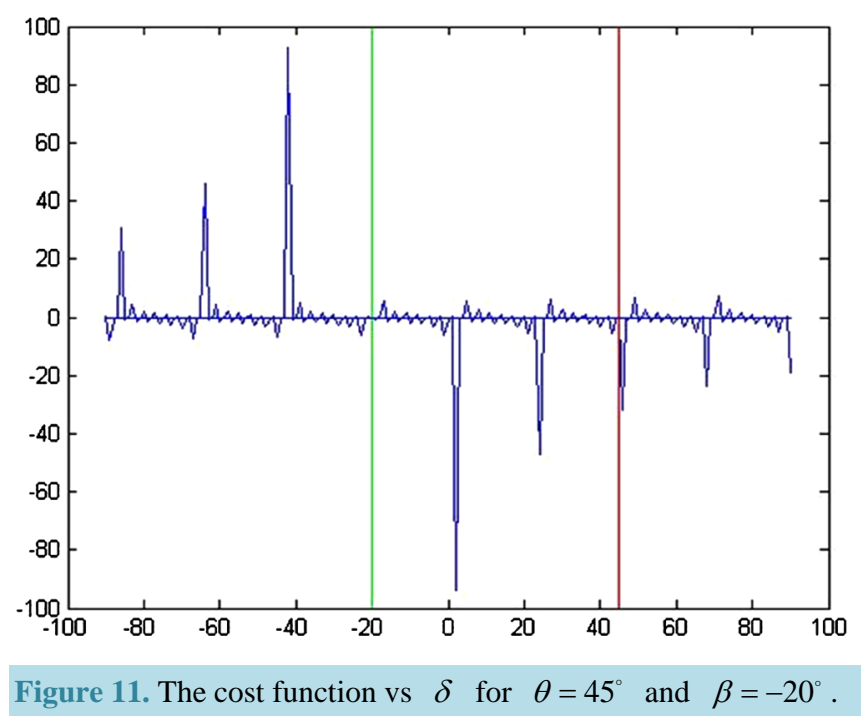


Using simulated annealing we solve the same example as shown in Figure 12. Here the global optimal $\delta$ is found to be at $18.66^{\circ}$ after 10 iterations.

In the second example for $\theta=45^{\circ}$ and $\beta=40^{\circ}$. Figure 13 shows a numerical calculation of the cost function given in Equation (17). A gain, the optimal $\delta$ has more than one solution at the zero crossing points one of them is the global minimum cost solution.

Using simulated annealing we solve the same example as shown in Figure 14. Here the global optimal $\delta$ is found to be at $-57.27^{\circ}$ after 6 iterations.

The simulated annealing algorithm in both examples arrives in few iterations at the global optimal solution. Next we discuss the results obtained for the whole devised system.

\section{Discussion of Results}

The proposed system uses two $\mathrm{H}$ shaped patch antennas with delay element to steer the radiation pattern of the resultant array in a way that ensures the safety of the user and at the same time maintain the connectivity with
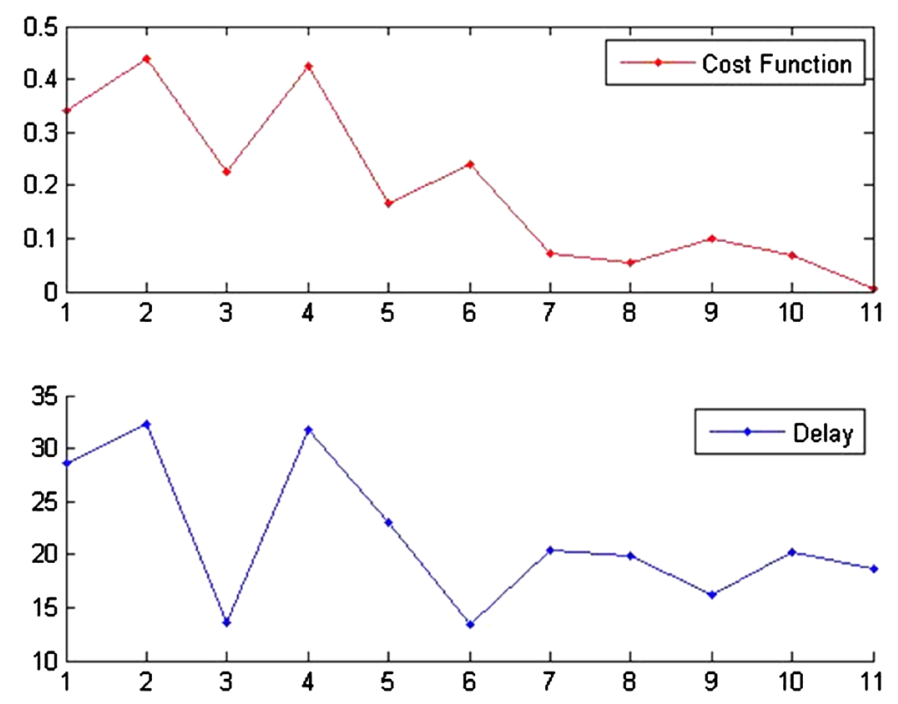

Figure 12. The cost function and $\delta$ vs iteration number for $\theta=45^{\circ}$ and $\beta=-20^{\circ}$.

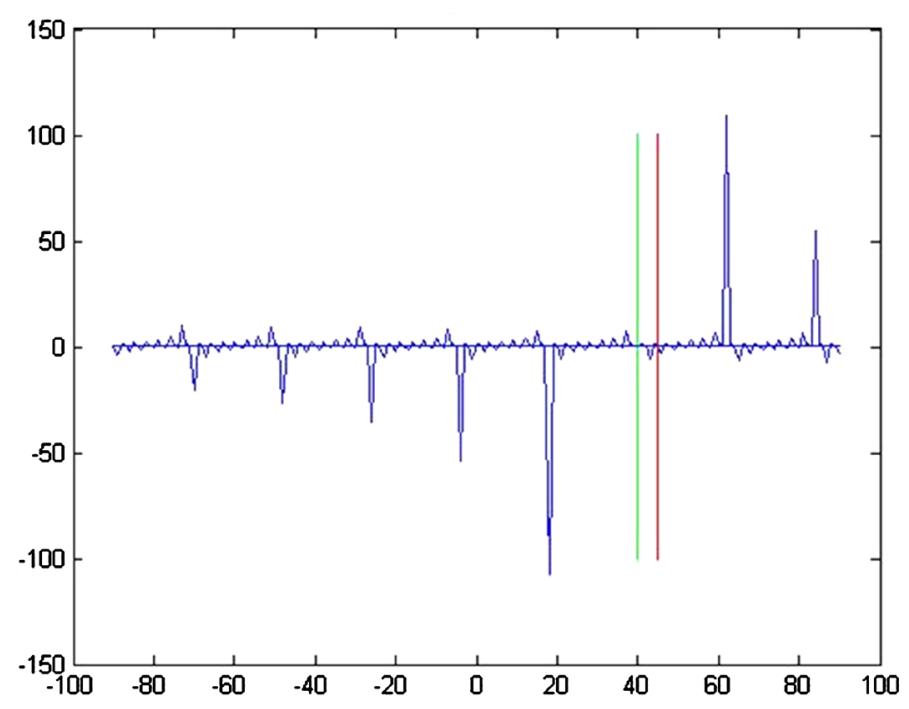

Figure 13. The cost function vs $\delta$ for $\theta=45^{\circ}$ and $\beta=40^{\circ}$. 

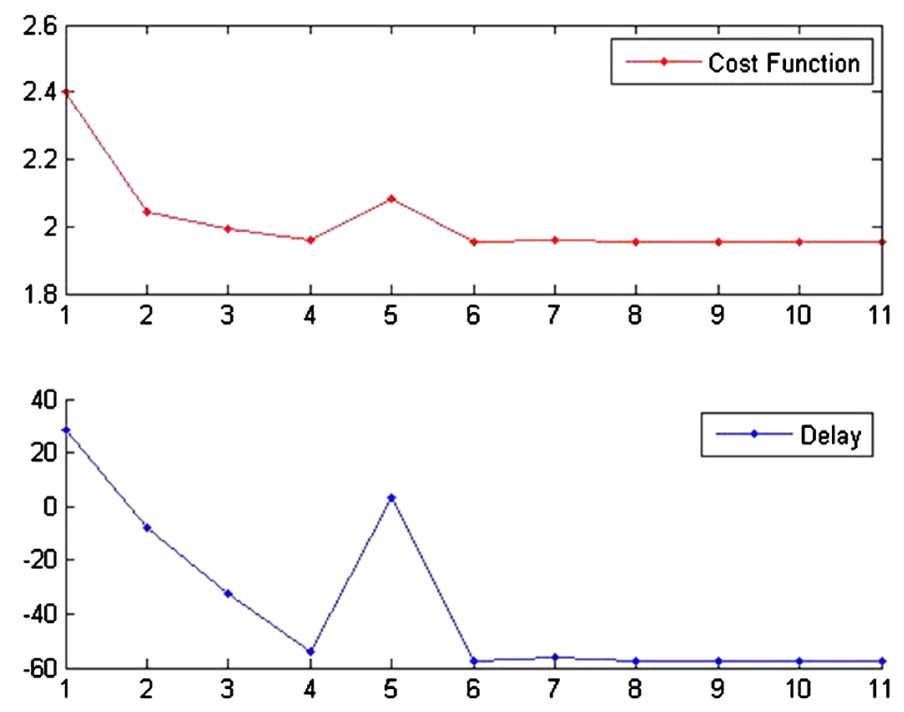

Figure 14. The cost function and $\delta$ vs iteration number for $\theta=45^{\circ}$ and $\beta=40^{\circ}$.

the cellular network. Maximizing the radiated power toward the base station while keeping the SAR level under the allowable maximum value is used as the optimization criteria. This problem is solved using Lagrange multiplier method and yields a numerically challenging solution; therefore, simulated annealing algorithm is used to find a sub-optimal solution that works fast in real time for the mobile unit. Numerical calculations showed good and efficient solutions for the optimal delay value when using the simulated annealing algorithm.

The design is simple and can be easily implemented on mobile units; it does not need large processing power from the mobile unit. It can be implemented in real time with minimum cost. Local field is also affects the user and needs to be investigated in future work.

\section{References}

[1] Hanus, X., Luong, M. and Lethimonnier, F. (2005) Electromagnetics Fields and SAR Computations in a Human Head with a Multi-Port Driven RF Coil at 11.7 Tesla. Proceedings of the International Society for Magnetic Resonance in Medicine, 13, 876.

[2] CENELEC, EN 62209 (2006) Human Exposure to Radio Frequency Fields from Hand-Held and Body-Mounted Wireless Communication Devices-Human Models, Instrumentation, and Procedures-Part 1: Procedure to Determine the Specific Absorption Rate (SAR) for Hand-Held Devices Used in Close Proximity to the Ear (Frequency Range of 300 $\mathrm{MHz}$ to $3 \mathrm{GHz}$ ).

[3] Gabriel, C. (2000) Dielectric Properties of Tissues. In: Klauenberg, B.J. and Miklavcic, D., Eds., RFR Dosimetry and Its Relationship to the Biological Effects of EMFs, NATO Science Series 3, 82, 75-84.

[4] Gabriel, C. and Peyman, A. (2006) Dielectric Measurement: Error Analysis and Assessment of Uncertainty. Physics in Medicine and Biology, 51, 6033-6046. http://dx.doi.org/10.1088/0031-9155/51/23/006

[5] IEC 62209-1 (2005) Human Exposure to Radio Frequency Fields from Hand-Held and Body-Mounted Wireless Communication Devices_-Human Models, Instrumentation, and Procedures_-Part 1: Procedure to Determine the Specific Absorption Rate (SAR) for Hand-Held Devices Used in Close Proximity to the Ear (Frequency Range of 300 $\mathrm{MHz}$ to $3 \mathrm{GHz})$.

[6] IEEE Std 1528T (2003) IEEE Recommended Practice for Determining the Peak Spatial—Average Specific Absorption Rate (SAR) in the Human Head from Wireless Communications Devices: Measurement Techniques.

[7] Kuster, N. and Balzano, Q. (1992) Energy Absorption Mechanism by Biological Bodies in the Near Field of Dipole Antennas above 300 MHz. IEEE Transactions on Vehicular Technology, 41, 17-23. http://dx.doi.org/10.1109/25.120141

[8] Kuster, N., Balzano, Q. and Lin, J. (1997) Mobile Communication Safety. Chapman and Hall, New York, 21-22.

[9] IEEE Std C95.1 ${ }^{\mathrm{TM}}$-2005 (2005) IEEE Standard for Safety Levels with Respect to Human Exposure to Radio Frequency Electromagnetic Fields, $3 \mathrm{kHz}$ to $300 \mathrm{GHz}$. 
[10] Schmid, T., Egger, O. and Kuster, N. (1996) Automated E-Field Scanning System for Dosimetric Assessments. IEEE Transactions on Microwave Theory and Techniques, 44, 105-113. http://dx.doi.org/10.1109/22.481392

[11] Gandhi, O., Lazzi, G. and Furse, C. (1996) EM Absorption in the Human Head Neck for Mobile Telephones at 835 and 1900 MHz. IEEE Transactions on Microwave Theory and Techniques, 44, 1884-1897.

[12] Dimbylow, P. and Mann, S. (1994) SAR Calculations in an Anatomically Realistic Model of the Head for Mobile Communication Transceivers at $900 \mathrm{MHz}$ and $1.8 \mathrm{GHz}$. Physics in Medicine and Biology, 39, 1537-1553. http://dx.doi.org/10.1088/0031-9155/39/10/003

[13] Berntsen, S. and Hornsleth, S. (1994) Retarded Time Absorbing Boundary Conditions. IEEE Transactions on Antenna and Propagation, 42, 1059-1064.

[14] Agarwal, A., Desai, N.R., Makker, K., Varghese, A., Mouradi, R., Sabanegh, E. and Sharma, R. (2009) Effects of Radiofrequency Electromagnetic Waves (RF-EMW) from Cellular Phones on Human Ejaculated Semen: An in Vitro Pilot Study. Fertility and Sterility, 92, 1318-1325. http://dx.doi.org/10.1016/j.fertnstert.2008.08.022

[15] BS EN 62209-1: (2006) Human Exposure to Radio Frequency Fields from Hand-Held and Body-Mounted Wireless Communication Devices-Human Models, Instrumentation, and Procedures-Part 1: Procedure to Determine the Specific Absorption Rate (SAR) for Hand-Held Devices Used in Close Proximity to the Ear (Frequency Range of 300 $\mathrm{MHz}$ to $3 \mathrm{GHz}$ ).

[16] Christodoulous, C. and Herscovicl, N. (2000) Smart Antennas in Wireless Communications: Base-Station Diversity and Handset Beamforming. IEEE Antennas and Propagation Magazine, 42, 142-151. http://dx.doi.org/10.1109/74.883513

[17] Rahhal, J. and Al-Nadi, D.A. (2007) A General Configuration Antenna Array for Multi-User Systems with Genetic and Ant Coloni Optimization. Journal of Electromagnetics, 27, 413-426. http://dx.doi.org/10.1080/02726340701572991

[18] Mouhamadou, M., Vaudon, P. and Rammal, M. (2006) Smart Antenna Array Patterns Synthesis: Null Steering and Multi-User Beamforming by Phase Control. Progress in Electromagnetics Research, 60, 95-106. http://dx.doi.org/10.2528/PIER05112801

[19] Ingber, L. (1993) Simulated Annealing: Practice versus Theory. Mathematical and Computer Modelling, 18, 29-57. http://dx.doi.org/10.1016/0895-7177(93)90204-C

[20] Johnson, D.S., Aragon, C.R., McGeoch, L.A. and Schevon, C. (1989) Optimization by Simulated Annealing: An Experimental Evaluation; Part I, Graph Partitioning. Operations Research, 37, 865-892.

[21] van Laarhoven, P.J.M. and Aarts, E.H.L. (1987) Simulated Annealing: Theory and Applications. Mathematics and Its Applications, 37, 7-15.

[22] Coleman, C.M., Rothwell, E.J. and Ross, J.E. (2004) Investigation of Simulated Annealing, Ant-Colony Optimization, and Genetic Algorithms for Self-Structuring Antennas. IEEE Transactions on Antennas and Propagation, 52, 10071014. 
Scientific Research Publishing (SCIRP) is one of the largest Open Access journal publishers. It is currently publishing more than 200 open access, online, peer-reviewed journals covering a wide range of academic disciplines. SCIRP serves the worldwide academic communities and contributes to the progress and application of science with its publication.

Other selected journals from SCIRP are listed as below. Submit your manuscript to us via either submit@scirp.org or Online Submission Portal.
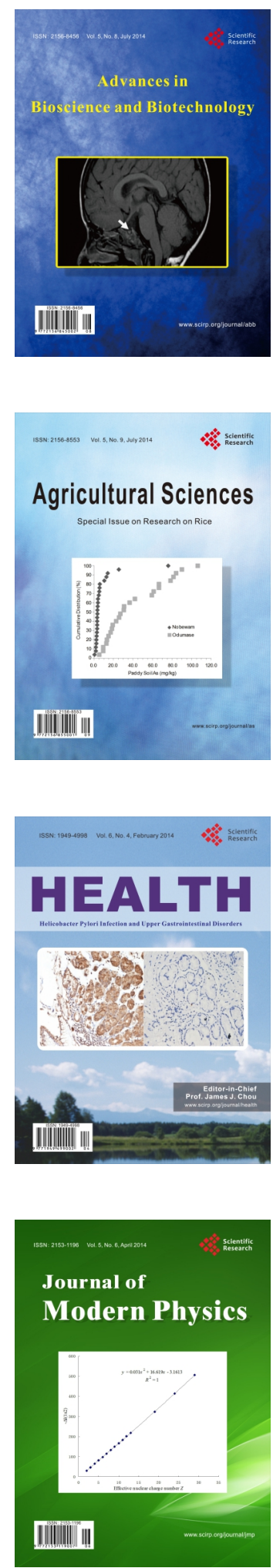
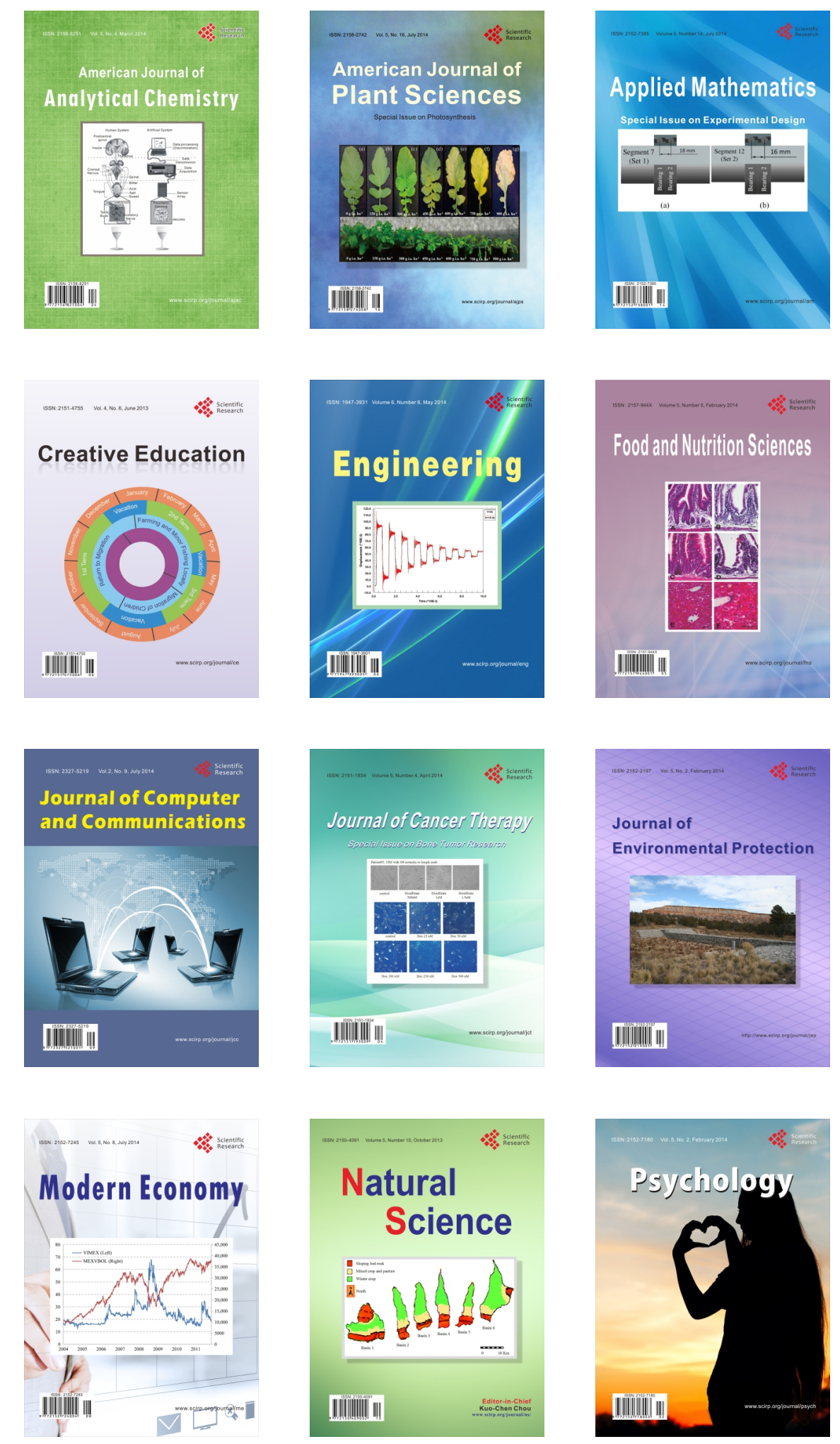